Pacific

Journal of

Mathematics

SUFFICIENT CONDITIONS FOR ROBUSTNESS OF ATTRACTORS

\author{
C.A. Morales and M.J. Pacifico
}




\title{
SUFFICIENT CONDITIONS FOR ROBUSTNESS OF ATTRACTORS
}

\author{
C.A. Morales And M.J. Pacifico
}

\begin{abstract}
A recent problem in dynamics is to determine whether an attractor $\Lambda$ of a $C^{r}$ flow $X$ is $C^{r}$ robust transitive. By an attractor we mean a transitive set to which all positive orbits close to it converge. An attractor is $C^{r}$ robust transitive (or $C^{r}$ robust for short) if it has a neighborhood $U$ such that the set $\bigcap_{t>0} Y_{t}(U)$ is transitive for every flow $Y$ that is $C^{r}$ close to $X$. We give sufficient conditions for robustness of attractors based on the following definitions: an attractor is singularhyperbolic if it has singularities, all of which are hyperbolic, and is partially hyperbolic with volume expanding central direction (Morales, Pacifico and Pujals, 1998). An attractor is $C^{r}$ critically robust if it has a neighborhood $U$ such that $\bigcap_{t>0} Y_{t}(U)$ is in the closure of the closed orbits of every flow $Y C^{r}$ close to $X$. We show that on compact 3-manifolds all $C^{r}$ critically robust singular-hyperbolic attractors with only one singularity are $C^{r}$ robust.
\end{abstract}

\section{Introduction}

A recent problem in dynamics is to determine whether an attractor $\Lambda$ of a $C^{r}$ flow $X$ is $C^{r}$ robust transitive or not. By an attractor we mean a transitive set to which all positive orbits close to it converge. An attractor is $C^{r}$ robust transitive (or $C^{r}$ robust for short) if it has a neighborhood $U$ such that the set $\bigcap_{t>0} Y_{t}(U)$ is transitive for every flow $Y$ that is $C^{r}$ close to $X$. We give sufficient conditions for robustness of attractors based on the following definitions: an attractor is singular-hyperbolic if it has singularities, all of which are hyperbolic, and is partially hyperbolic with volume expanding central direction $[\mathrm{MPP}]$. An attractor is $C^{r}$ critically robust if it has a neighborhood $U$ such that $\bigcap_{t>0} Y_{t}(U)$ is in the closure of the closed orbits is every flow $Y C^{r}$ close to $X$. We show that, for flows on compact 3manifolds, all $C^{r}$ critically robust singular-hyperbolic attractors with only one singularity are $C^{r}$ robust.

Let us state our result in a precise way. Hereafter $X_{t}$ is a flow induced by a $C^{r}$ vector field $X$ on a compact 3-manifold $M$. The $\omega$-limit set of $p \in M$ is the accumulation point set $\omega_{X}(p)$ of the positive orbit of $p$. An invariant set is transitive if it equals $\omega_{X}(p)$ for some point $p$ on it. 
Definition 1.1. A compact set in $M$ is an attracting set of $X$ if it can be written in the form $\bigcap_{t>0} X_{t}(U)$ for some neighborhood $U$. An attractor is a transitive attracting set.

See $[\mathbf{M i}]$ where several definitions of attractors are discussed. The central definition of this paper is the following:

Definition 1.2. An attractor of a $C^{r}$ flow $X$ is $C^{r}$ robust transitive (or $C^{r}$ robust for short) if it has a neighborhood $U$ such that $\bigcap_{t>0} Y_{t}(U)$ is a transitive set of $Y$ for every flow $Y$ that is $C^{r}$ close to $X$.

Recently the problem of finding sufficient conditions for robustness of attractors was introduced in $[\mathbf{B}]$ and $[\mathbf{P}]$. To deal with it we introduce the following definitions: a compact invariant set $\Lambda$ of $X$ is partially hyperbolic if there are an invariant splitting $T \Lambda=E^{s} \oplus E^{c}$ and positive constants $K, \lambda$ such that:

1. $E^{s}$ is contracting, namely

$$
\left\|D X_{t} / E_{x}^{s}\right\| \leq K e^{-\lambda t}, \quad \forall x \in \Lambda, \forall t>0 .
$$

2. $E^{s}$ dominates $E^{c}$, namely

$$
\left\|D X_{t} / E_{x}^{s}\right\| \cdot\left\|D X_{-t} / E_{X_{t}(x)}^{c}\right\| \leq K e^{-\lambda t}, \quad \forall x \in \Lambda, \forall t>0 .
$$

The central direction $E^{c}$ of $\Lambda$ is said to be volume expanding if the additional condition

$$
\left|J\left(D X_{t} / E_{x}^{c}\right)\right| \geq K e^{\lambda t}
$$

holds for all $x \in \Lambda$ and $t>0$, where $J(\cdot)$ means the Jacobian.

Definition 1.3 ([MPP]). An attractor is singular-hyperbolic if it has singularities, all of which are hyperbolic, and is partially hyperbolic with volume expanding central direction.

The most representative example of a $C^{r}$ robust singular-hyperbolic attractor is the geometric Lorenz attractor $[\mathbf{G W}]$. The main result in $[\mathbf{M P P}]$ claims that $C^{1}$ robust nontrivial attractors on compact 3 -manifolds are singular-hyperbolic. The converse is false: there are singular-hyperbolic attractors on compact 3 -manifolds that are not $C^{r}$ robust [MPu]. The following definition gives a further sufficient condition for robustness of singularhyperbolic attractors:

Definition 1.4. An attractor of a $C^{r}$ flow $X$ is $C^{r}$ critically robust if it has a neighborhood $U$ such that $\bigcap_{t>0} Y_{t}(U)$ is in the closure of the closed orbits of $Y$, for every flow $Y$ that is $C^{r}$ close to $X$.

Hyperbolic attractors on compact manifolds are $C^{r}$ robust and $C^{r}$ critically robust for all $r$. The geometric Lorenz attractor $[\mathbf{G W}]$ is an example of a singular-hyperbolic attractor with only one singularity which is also $C^{r}$ robust and $C^{r}$ critically robust. In general singular-hyperbolic attractors 
with only one singularity may be neither $C^{r}$ robust nor $C^{r}$ critically robust [MPu]. Nevertheless we shall prove that on compact 3-manifolds $C^{r}$ critically robustness implies $C^{r}$ robustness among singular-hyperbolic attractors with only one singularity. More precisely:

Theorem A. A $C^{r}$ critically robust singular-hyperbolic attractor with only one singularity on compact 3-manifolds is $C^{r}$ robust.

This gives explicit sufficient conditions for the robustness of attractors but they depend on the perturbed flow. E. Pujals is interested in conditions depending on the unperturbed flow only. It would also be interesting to determine whether the conclusion of Theorem A holds if we interchange the roles of robust and critically robust in the statement.

The proof of Theorem A relies on recent work [MP2]. We reproduce the necessary results in Section 2 for completeness. The proof of Theorem A is in Section 3.

\section{Singular-hyperbolic attracting sets}

In this section we describe the results in [MP2], omitting some proofs; see [MP2] for details. Hereafter $X$ is a $C^{r}$ flow on a closed 3-manifold $M$. The closure of $B$ will be denoted by $\mathrm{Cl}(B)$. If $A$ is a compact invariant set of $X$ we denote by $\operatorname{Sing}_{X}(A)$ the set of singularities of $X$ in $A$, and by $\operatorname{Per}_{X}(A)$ the union of the periodic orbits of $X$ in $A$. A compact invariant set $H$ of $X$ is hyperbolic if the tangent bundle over $H$ has an invariant decomposition $E^{s} \oplus E^{X} \oplus E^{u}$ such that $E^{s}$ is contracting, $E^{u}$ is expanding and $E^{X}$ is generated by the direction of $X[\mathbf{P T}]$. Stable Manifold Theory [HPS] asserts the existence of the stable manifold $W_{X}^{s}(p)$ and the unstable manifold $W_{X}^{u}(p)$ associated to $p \in H$. These manifolds are respectively tangent to the subspaces $E_{p}^{s} \oplus E_{p}^{X}$ and $E_{p}^{X} \oplus E_{p}^{u}$ of $T_{p} M$. In particular, $W_{X}^{s}(p)$ and $W_{X}^{u}(p)$ are well-defined if $p$ belongs to a hyperbolic periodic orbit of $X$. If $O$ is an orbit of $X$ we write $W_{X}^{s}(O)=W_{X}^{s}(p)$ and $W_{X}^{u}(O)=$ $W_{X}^{u}(p)$ for some $p \in O$. We observe that $W_{X}^{s(u)}(O)$ does not depend on $p \in O$. When $\operatorname{dim} E^{s}=\operatorname{dim} E^{u}=1$ we say that $H$ is of saddle type. In this case $W_{X}^{s}(p)$ and $W_{X}^{u}(p)$ are two-dimensional submanifolds of $M$. The maps $p \in H \rightarrow W_{X}^{s}(p)$ and $p \in H \rightarrow W_{X}^{u}(p)$ are continuous (on compact parts). Moreover, a compact, singular, invariant set $\Lambda$ of $X$ is singularhyperbolic if all its singularities are hyperbolic and the tangent bundle over $\Lambda$ has an invariant decomposition $E^{s} \oplus E^{c}$ such that $E^{s}$ is contracting, $E^{s}$ dominates $E^{c}$ and $E^{c}$ is volume expanding (i.e., the Jacobian of $D X_{t} / E^{c}$ grows exponentially as $t \rightarrow \infty)$. Again, Stable Manifold Theory asserts the existence of the strong stable manifold $W_{X}^{s s}(p)$ associated to $p \in \Lambda$. This manifold is tangent to the subspace $E_{p}^{s}$ of $T_{p} M$. For all $p \in \Lambda$ we define $W_{X}^{s}(p)=\bigcup_{t \in \mathbf{R}} W_{X}^{s s}\left(X_{t}(p)\right)$. If $p$ is regular (i.e., $X(p) \neq 0$ ) then $W_{X}^{s}(p)$ is a 
well-defined two-dimensional submanifold of $M$. The map $p \in \Lambda \rightarrow W_{X}^{s}(p)$ is continuous (on compact parts) at the regular points $p$ of $\Lambda$. A singularity $\sigma$ of $X$ is Lorenz-like if its eigenvalues $\lambda_{1}, \lambda_{2}, \lambda_{3}$ are real and satisfy

$$
\lambda_{2}<\lambda_{3}<0<-\lambda_{3}<\lambda_{1}
$$

up to some reordering of the eigenvalues. A Lorenz-like singularity $\sigma$ is hyperbolic, so $W_{X}^{s}(\sigma)$ and $W_{X}^{u}(\sigma)$ do exist. Moreover, the eigenspace of $\lambda_{2}$ is tangent to a one-dimensional invariant manifold $W_{X}^{s s}(\sigma)$. This manifold is called the strong stable manifold of $\sigma$. Clearly $W_{X}^{s s}(\sigma)$ splits $W_{X}^{s}(\sigma)$ into two connected components. We denote by $W_{X}^{s,+}(\sigma)$ and $W_{X}^{s,-}(\sigma)$ the two connected components of $W_{X}^{s}(\sigma) \backslash W_{X}^{s s}(\sigma)$.

Let $\Lambda$ be a singular-hyperbolic set with dense periodic orbits of a threedimensional flow. It follows from $[\mathbf{M P P}]$ that every $\sigma \in \operatorname{Sing}_{X}(\Lambda)$ is Lorenzlike and satisfies $\Lambda \cap W_{X}^{s s}(\sigma)=\{\sigma\}$. It follows also from [MPP] that any compact invariant subset without singularities of $\Lambda$ is hyperbolic of saddle type. If in addition $\Lambda$ is attracting, there is for every $p \in \operatorname{Per}_{X}(\Lambda)$ a $\sigma \in \operatorname{Sing}_{X}(\Lambda)$ such that

$$
W_{X}^{u}(p) \cap W_{X}^{s}(\sigma) \neq \emptyset .
$$

This follows from the methods in [MP1].

For every singular-hyperbolic set $\Lambda$ of a three-dimensional flow $X$ and every Lorenz-like singularity $\sigma \in \operatorname{Sing}_{X}(\Lambda)$ we define

$$
\begin{aligned}
& P^{+}=\left\{p \in \operatorname{Per}_{X}(\Lambda): W_{X}^{u}(p) \cap W_{X}^{s,+}(\sigma) \neq \emptyset\right\}, \\
& P^{-}=\left\{p \in \operatorname{Per}_{X}(\Lambda): W_{X}^{u}(p) \cap W_{X}^{s,-}(\sigma) \neq \emptyset\right\}, \\
& H_{X}^{+}=\operatorname{Cl}\left(P^{+}\right), \\
& H_{X}^{-}=\operatorname{Cl}\left(P^{-}\right) .
\end{aligned}
$$

These sets will play an important role.

Lemma 2.1. Let $\Lambda$ be a connected, singular-hyperbolic, attracting set with dense periodic orbits and only one singularity $\sigma$. Then $\Lambda=H_{X}^{+} \cup H_{X}^{-}$.

Next we state a technical lemma to be used later. If $S$ is a submanifold we denote by $T_{x} S$ the tangent space at $x \in S$. A cross-section of $X$ is a compact submanifold $\Sigma$ transverse to $X$ and diffeomorphic to the two-dimensional square $[0,1]^{2}$. If $\Lambda$ is a singular-hyperbolic set of $X$ and $x \in \Sigma \cap \Lambda$, then $x$ is regular and so $W_{X}^{s}(x)$ is a two-dimensional submanifold transverse to $\Sigma$. In this case we denote by $W_{X}^{s}(x, \Sigma)$ the connected component of $W_{X}^{s}(x) \cap \Sigma$ containing $x$. We shall be interested in a special cross-section described as follows: let $\Lambda$ be a singular-hyperbolic set of a three-dimensional flow $X$ and let $\sigma \in \operatorname{Sing}_{X}(\Lambda)$. Suppose that the closed orbits contained in $\Lambda$ are dense in $\Lambda$. Then $\sigma$ is Lorenz-like [MPP], and therefore one can describe the flow using the Grobman-Hartman Theorem [dMP]. Indeed, we can assume that the flow of $X$ around $\sigma$ is the linear flow $\lambda_{1} \partial_{x_{1}}+\lambda_{2} \partial_{x_{2}}+\lambda_{3} \partial_{x_{3}}$ 
in a suitable coordinate system $\left(x_{1}, x_{2}, x_{3}\right) \in[-1,1]^{3}$ around $\sigma=(0,0,0)$. A cross-section $\Sigma$ of $X$ is singular if it corresponds to the submanifolds $\Sigma^{+}=\left\{x_{3}=1\right\}$ or $\Sigma^{-}=\left\{x_{3}=-1\right\}$ in the coordinate system $\left(x_{1}, x_{2}, x_{3}\right)$. We denote by $l^{+}$and $l^{-}$the curves obtained by intersecting $\left\{x_{2}=0\right\}$ with $\Sigma^{+}$and $\Sigma^{-}$, respectively; these curves are contained in $W_{X}^{s,+}(\sigma)$ and $W_{X}^{s,-}(\sigma)$ respectively. We state without proof the following straightforward lemma:

Lemma 2.2. Let $\Lambda$ a singular-hyperbolic set with dense periodic orbits of a three-dimensional flow $X$, and fix $\sigma \in \operatorname{Sing}_{X}(\Lambda)$. There are singular cross-sections $\Sigma^{+}, \Sigma^{-}$as above such that every orbit of $\Lambda$ passing close to some point in $W_{X}^{s,+}(\sigma)$ (resp. $\left.W_{X}^{s,-}(\sigma)\right)$ intersects $\Sigma^{+}\left(\right.$resp. $\left.\Sigma^{-}\right)$. If $p \in$ $\Lambda \cap \Sigma^{+}$is close to $l^{+}$, then $W_{X}^{s}\left(p, \Sigma^{+}\right)$is a vertical curve crossing $\Sigma^{+}$. If $p \in \operatorname{Per}_{X}(\Lambda)$ and $W_{X}^{u}(p) \cap W_{X}^{s,+}(\sigma) \neq \emptyset$, then $W_{X}^{u}(p)$ contains an interval $J=J_{p}$ intersecting $l^{+}$transversally; and the same is true if we replace + by - .

To prove transitivity we shall use two lemmas:

Lemma 2.3 (Birkhoff's criterion). Let $T$ be a compact, invariant set of $X$ such that for all open sets $U, V$ intersecting $T$ there is $s>0$ such that $X_{s}(U \cap T) \cap V \neq \emptyset$. Then $T$ is transitive.

Lemma 2.4. Let $\Lambda$ be a connected, singular-hyperbolic, attracting set with dense periodic orbits and only one singularity $\sigma$. Let $U, V$ be open sets, $p \in U \cap \operatorname{Per}_{X}(\Lambda)$ and $q \in V \cap \operatorname{Per}_{X}(\Lambda)$. If $W_{X}^{u}(p) \cap W_{X}^{s,+}(\sigma) \neq \emptyset$ and $W_{X}^{u}(q) \cap W_{X}^{s,+}(\sigma) \neq \emptyset$, there exist $t>0$ and $z \in W_{X}^{u}(p)$ arbitrarily close to $W_{X}^{u}(p) \cap W_{X}^{s,+}(\sigma)$ such that $X_{t}(z) \in V$. The same is true if we replace + by

Let $\Lambda$ be a singular-hyperbolic set of $X \in \mathcal{X}^{r}$ satisfying:

1. $\Lambda$ is connected.

2. $\Lambda$ is attracting.

3. The closed orbits contained in $\Lambda$ are dense in $\Lambda$.

4. $\Lambda$ has only one singularity $\sigma$.

We note that condition 3 implies

$$
\Lambda=\mathrm{Cl}\left(\operatorname{Per}_{X}(\Lambda)\right) \text {. }
$$

Proposition 2.5. Suppose that, for any given $p, q \in \operatorname{Per}_{X}(\Lambda)$, either

1. $W_{X}^{u}(p) \cap W_{X}^{s,+}(\sigma) \neq \emptyset$ and $W_{X}^{u}(q) \cap W_{X}^{s,+}(\sigma) \neq \emptyset$, or

2. $W_{X}^{u}(p) \cap W_{X}^{s,-}(\sigma) \neq \emptyset$ and $W_{X}^{u}(q) \cap W_{X}^{s,-}(\sigma) \neq \emptyset$.

Then $\Lambda$ is transitive.

Proof. By Birkhoff's criterion we only need prove that for all open sets $U, V$ intersecting $\Lambda$ there exists $s>0$ such that $X_{s}(U \cap \Lambda) \cap V \neq \emptyset$. For this we proceed as follows: by (H1) there are $p \in \operatorname{Per}_{X}(\Lambda) \cap U$ and $q \in \operatorname{Per}_{X}(\Lambda) \cap V$. 
First suppose that alternative 1 holds. By Lemma 2.4, there are $z \in W_{X}^{u}(p)$ and $t>0$ such that $X_{t}(z) \in V$. Since $z \in W_{X}^{u}(p)$, we have $w=X_{-t^{\prime}}(z) \in U$ for some $t^{\prime}>0$. Since $\Lambda$ is an attracting set, $w \in \Lambda$. If $s=t+t^{\prime}>0$ we conclude that $w \in(U \cap \Lambda) \cap X_{-s}(V)$ and so $X_{s}(U \cap \Lambda) \cap V \neq \emptyset$. If alternative 2 of the corollary holds we can find $s>0$ such that $X_{s}(U \cap \Lambda) \cap V \neq \emptyset$ in a similar way (replacing + by - ). The result follows.

Proposition 2.6. If there is a sequence $p_{n} \in \operatorname{Per}_{X}(\Lambda)$ converging to some point in $W_{X}^{s,+}(\sigma)$ such that $W_{X}^{u}\left(p_{n}\right) \cap W_{X}^{s,-}(\sigma) \neq \emptyset$ for all $n$, then $\Lambda$ is transitive. The same is true interchanging + and - .

Proof. Let $p, q \in \operatorname{Per}_{X}(\Lambda)$ be fixed. Suppose that $W_{X}^{u}(p) \cap W_{X}^{s,+}(\sigma) \neq \emptyset$ and $W_{X}^{u}(q) \cap W_{X}^{u,-}(\sigma) \neq \emptyset$. By Lemma 2.2 we can fix a cross-section $\Sigma=\Sigma^{+}$ through $W_{X}^{s,+}(\sigma)$ and an open arc $J \subset \Sigma \cap W_{X}^{u}(p)$ intersecting $W_{X}^{s,+}(\sigma)$ transversally. Again by Lemma 2.2 we can assume that $p_{n} \in \Sigma$ for every $n$. Because the direction $E^{s}$ of $\Lambda$ is contracting, the size of $W_{X}^{s}\left(p_{n}\right)$ is uniformly bounded away from zero. It follows that there is $n$ large so that $J$ intersects $W_{X}^{S}\left(p_{n}\right)$ transversally. Applying the Inclination Lemma [dMP] to the saturation of $J \subset W_{X}^{u}(p)$, and the assumption $W_{X}^{u}\left(p_{n}\right) \cap W_{X}^{s,-}(\sigma) \neq \emptyset$, we conclude that $W_{X}^{u}(p) \cap W_{X}^{s,-}(\sigma) \neq \emptyset$. So alternative 2 of Proposition 2.5 holds; it follows from that proposition that $\Lambda$ is transitive.

Proposition 2.7. If there is $a \in W_{X}^{u}(\sigma) \backslash\{\sigma\}$ such that $\sigma \in \omega_{X}(a)$, then $\Lambda$ is transitive.

Proof. Without loss of generality we can assume that there exists $z$ in $\omega_{X}(a) \cap W_{X}^{+}(\sigma)$. If $W_{X}^{u}(q) \cap W_{X}^{s,-}(\sigma)=\emptyset$ for all $q \in \operatorname{Per}_{X}(\Lambda)$, then $W_{X}^{u}(q) \cap W_{X}^{s,+}(\sigma) \neq \emptyset$ for all $q \in \operatorname{Per}_{X}(\Lambda)$; see [MP1]. Then $\Lambda$ is transitive by Proposition 2.5 since alternative 1 holds for all $p, q \in \operatorname{Per}_{X}(\Lambda)$. So we can assume that there is $q \in \operatorname{Per}_{X}(\Lambda)$ such that $W_{X}^{u}(q) \cap W_{X}^{s,-}(\sigma) \neq \emptyset$. It follows from the dominating condition of the singular-hyperbolic splitting of $\Lambda$ that the intersection $W_{X}^{u}(q) \cap W_{X}^{s,-}(\sigma)$ is transversal. This allows us to choose a point in $W_{X}^{u}(q)$ arbitrarily close to $W_{X}^{s,-}(\sigma)$ on the side of $W_{X}^{u}(q) \cap W_{X}^{s,-}(\sigma)$ accumulating $a$. Since $\Lambda$ is attracting and satisfies (H1), one can find a sequence $p_{n} \in \operatorname{Per}_{X}(\Lambda)$ converging to $z \in W_{X}^{s,+}(\sigma)$ such that for all $n$ there is $p_{n}^{\prime}$ in the orbit of $p_{n}$ such that the sequence $p_{n}^{\prime}$ converges to some point in $W_{X}^{s,-}(\sigma)$. Now suppose for a contradiction that $\Lambda$ is not transitive. Then Proposition 2.6 implies

$$
W_{X}^{u}\left(p_{n}^{\prime}\right) \cap W_{X}^{s,+}(\sigma)=\emptyset \quad \text { and } \quad W_{X}^{u}\left(p_{n}\right) \cap W_{X}^{s,-}(\sigma)=\emptyset
$$

for $n$ large. But $W_{X}^{u}\left(p_{n}\right)=W_{X}^{u}\left(p_{n}^{\prime}\right)$ since $p_{n}^{\prime}$ and $p_{n}$ are in the same orbit of $X$. So $W_{X}^{u}\left(p_{n}\right) \cap\left(W_{X}^{s,+}(\sigma) \cup W_{X}^{s,-}(\sigma)\right)=\emptyset$. However

$$
W_{X}^{u}\left(p_{n}\right) \cap W_{X}^{s}(\sigma)=\emptyset
$$

a contradiction since $\operatorname{Sing}_{X}(\Lambda)=\{\sigma\}$. We conclude that $\Lambda$ is transitive. 
Theorem 2.8. If $\Lambda$ is not transitive, there is for all $a \in W_{X}^{u}(\sigma) \backslash\{\sigma\}$ a periodic orbit $O$ of $X$ with positive expanding eigenvalues such that $a \in W_{X}^{s}(O)$.

Proof. Fix $a \in W_{X}^{u}(\sigma) \backslash\{\sigma\}$, and assume that $\omega_{X}(a)$ is not a periodic orbit. We will obtain a contradiction once we prove that if $p, q \in \operatorname{Per}_{X}(\Lambda)$ then $p, q$ satisfy one of the two alternatives in Proposition 2.5. To prove this we proceed as follows: as noted before, both $W_{X}^{u}(p)$ and $W_{X}^{u}(q)$ intersect $W_{X}^{s}(\sigma)$ (see $\left.[\mathbf{M P 1}]\right)$. Then we can assume

$$
W_{X}^{u}(p) \cap W_{X}^{s,+}(\sigma) \neq \emptyset \quad \text { and } \quad W_{X}^{u}(q) \cap W_{X}^{s,-}(\sigma) \neq \emptyset .
$$

By using this and the linear coordinate around $\sigma$, it is easy to construct an open interval $I=I_{a}$, contained in a suitable cross-section $\Sigma=\Sigma_{a}$ of $X$ containing $a$, and such that $I \backslash\{a\}$ is formed by two intervals $I^{+} \subset W_{X}^{u}(p)$ and $I^{-} \subset W_{X}^{u}(q)$. Observe that the tangent vector of $I$ is contained in $E^{c} \cap T \Sigma_{a}$. Proposition 2.7 implies that $\sigma \notin \omega_{X}(a)$, since $\Lambda$ is not transitive. It follows that $H=\omega_{X}(a)$ is a hyperbolic set of saddle type; see [MPP]. As in $[\mathbf{M}]$ one proves that $H$ is one-dimensional, so Bowen's Theory of hyperbolic one-dimensional sets $[\mathbf{B w}]$ applies. In particular we can choose a family of cross-sections $\mathcal{S}=\left\{S_{1}, \ldots, S_{r}\right\}$ of small diameter such that $H$ is the flow-saturate of $H \cap \operatorname{int} \mathcal{S}^{\prime}$, where $\mathcal{S}^{\prime}=\bigcup S_{i}$ and int $\mathcal{S}^{\prime}$ is the interior of $\mathcal{S}^{\prime}$. Also, $I \subset \Lambda$ since $\Lambda$ is attracting. Recall that the tangent direction of $I$ is contained in $E^{c}$. Since $E^{c}$ is volume expanding and $H$ is nonsingular, the Poincaré map induced by $X$ on $\mathcal{S}^{\prime}$ is expanding along $I$. As in [MP1, p. 371] we can find $\delta>0$ and a open arc sequence $J_{n} \subset \mathcal{S}^{\prime}$ in the positive orbit of $I$ with length bounded away from 0 such that there is $a_{n}$ in the positive orbit of a contained in the interior of $J_{n}$. We can fix $S=S_{i} \in \mathcal{S}$ in order to assume that $J_{n} \subset S$ for every $n$. Let $x \in S$ be a limit point of $a_{n}$. Then $x \in H \cap \operatorname{int} \mathcal{S}^{\prime}$. Because $I$ is tangent to $E^{c}$, the interval sequence $J_{n}$ converges to an interval $J \subset W_{X}^{u}(x)$ in the $C^{1}$ topology $\left(W_{X}^{u}(x)\right.$ exists since $x \in H$ and $H$ is hyperbolic). $J$ is not trivial since the length of $J_{n}$ is bounded away from 0 . If $a_{n}$ were in $W_{X}^{s}(x)$ for $n$ large we would conclude that $x$ is periodic by [MP1, Lemma 5.6], a contradiction since $\omega_{X}(a)$ is not periodic. We conclude that for infinitely many values of $n, a_{n} \notin W_{X}^{s}(x)$. Since $J_{n} \rightarrow J$ and $\Lambda$ has strong stable manifolds of uniformly size, there exists

$$
z_{n} \in\left(W_{X}^{s}\left(a_{n+1}\right) \cap S\right) \cap\left(J_{n} \backslash\left\{a_{n}\right\}\right)
$$

for all $n$ large. For every $n$ let $J_{n}^{+}$and $J_{n}^{-}$be the two connected components of $J_{n} \backslash\left\{a_{n}\right\}$, with $J_{n}^{+}$in the positive orbit of $I^{+}$and $J_{n}^{-}$in the positive orbit of $I^{-}$. Clearly, either $z_{n} \in J_{n}^{+}$or $z_{n} \in J_{n}^{-}$.

If $z_{n} \in J_{n}^{+}$there is $v_{n+1} \in \operatorname{Per}_{X}(\Lambda) \cap S$ close to $a_{n+1}$ such that

$$
W_{X}^{s}\left(v_{n+1}\right) \cap J_{n}^{+} \neq \emptyset \quad \text { and } \quad W_{X}^{s}\left(v_{n+1}\right) \cap J_{n+1}^{-} \neq \emptyset .
$$

Since $v_{n+1}$ is periodic, it follows from [MPP] that $W_{X}^{u}\left(v_{n+1}\right)$ intersects $W_{X}^{s,+}(\sigma)$ or $W_{X}^{s,-}(\sigma)$. The choice of $v_{n+1}$ implies that its orbit passes close 
to a point in $W_{X}^{s,-}(\sigma)$. Since $\Lambda$ is not transitive we conclude that $W_{X}^{u}\left(v_{n+1}\right)$ intersects $W_{X}^{s,-}(\sigma)$. Since $W_{X}^{s}\left(v_{n+1}\right) \cap J_{n}^{+}$is transversal, the Inclination Lemma then implies $W_{X}^{u}(p) \cap W_{X}^{s,-}(\sigma) \neq \emptyset$. Hence

$$
W_{X}^{u}(p) \cap W_{X}^{s,-}(\sigma) \neq \emptyset \quad \text { and } \quad W_{X}^{u}(q) \cap W_{X}^{s,-}(\sigma) \neq \emptyset .
$$

If $z_{n} \in J_{n}^{-}$we can prove by similar arguments that

$$
W_{X}^{u}(p) \cap W_{X}^{s,+}(\sigma) \neq \emptyset \quad \text { and } \quad W_{X}^{u}(q) \cap W_{X}^{s,+}(\sigma) \neq \emptyset .
$$

These alternatives yield the desired contradiction. Therefore $\omega_{X}(a)=O$ for some periodic orbit $O$ of $X$. To finish we prove that the expanding eigenvalue of $O$ is positive. Suppose by contradiction that it is not. Fix a cross-section $\Sigma$ intersecting $O$ in a single point $p_{0}$. This section defines a Poincaré map $\Pi:$ Dom $\Pi \subset \Sigma \rightarrow \Sigma$ of which $p_{0}$ is a hyperbolic fixed-point. The assumption implies that $D \Pi\left(p_{0}\right)$ has negative expanding eigenvalue. Because $p_{0} \in \operatorname{Per}_{X}(\Lambda)$, it follows from $[\mathbf{M P P}]$ that $W_{X}^{u}\left(p_{0}\right)$ intersects $W_{X}^{s,+}(\sigma)$ or $W_{X}^{s,-}(\sigma)$. We shall assume the former case since the proof in the latter is similar. We claim that $W_{X}^{u}(p) \cap W_{X}^{s,+}(\sigma) \neq \emptyset$ for all $p \in \operatorname{Per}_{X}(\Lambda)$. Indeed, let $p \in \operatorname{Per}_{X}(\Lambda)$ be fixed. Again $W_{X}^{u}(p)$ intersects $W_{X}^{s,+}(\sigma)$ or $W_{X}^{s,-}(\sigma)$. In the first case we are done. So we can assume that $W_{X}^{u}(p) \cap W_{X}^{s,-}(\sigma) \neq \emptyset$. By flow-saturating this intersection we obtain an interval $K \subset W_{X}^{u}(p) \cap \Sigma$ intersecting $W_{X}^{s}\left(p_{0}, \Sigma\right)$ transversally. At the same time, there is an interval $J \subset W_{X}^{s,+}(\sigma) \cap \Sigma$ intersecting $W_{X}^{u}\left(p_{0}, \Sigma\right)$ transversally. Since the expanding eigenvalue of $D \Pi\left(p_{0}\right)$ is negative the Inclination Lemma implies that the backward iterates $\Pi^{-n}(J)$ of $J$ accumulate on $W_{X}^{s}\left(p_{0}, \Sigma\right)$ in both sides. Because $K$ has transversal intersection with $W_{X}^{s}\left(p_{0} \Sigma\right)$ we conclude that one such backward iterate intersects $K$, and this yields $W_{X}^{u}(p) \cap W_{X}^{s,+}(\sigma) \neq \emptyset$ as desired, proving the claim. The claim together with Proposition 2.5 implies that $\Lambda$ is transitive, yielding the contradiction needed to complete the proof of theorem.

Hereafter we shall assume that $\Lambda$ is not transitive. Let $a \in W_{X}^{s}(\sigma) \backslash\{\sigma\}$ be fixed. By Theorem 2.8, $a \in W_{X}^{s}(O)$ for some periodic orbit $O$ with positive expanding eigenvalue. This last property implies that the unstable manifold $W_{X}^{u}(O)$ of $O$ is a cylinder with generating curve $O$. Then $O$ separates $W_{X}^{u}(O)$ into two connected components, which we denote by $W^{u,+}, W^{u,-}$ according to the following convention (see Figure 1): there is an interval $I=I_{a}$, contained in a suitable cross-section of $X$ and containing $a$, such that if $I^{+}, I^{-}$are the connected components of $I \backslash\{a\}$ then $I^{+} \subset W_{X}^{u}(p)$ and $I^{-} \subset W_{X}^{u}(q)$ for some periodic points $p, q \in \Lambda$ (recall that $\Lambda$ is not transitive). In addition $I$ is tangent to the central direction $E^{c}$ of $\Lambda$ (see Figure 1). Since $a \in W_{X}^{s}(O)$ and $I$ is tangent to $E^{c}$, the flow of $X$ carries $I$ to an interval $I^{\prime}$ transverse to $W_{X}^{s}(O)$ at $a$. Note that the flow carries $I^{+}$ and $I^{-}$into $I_{0}^{+}$and $I_{0}^{-}$respectively. 
Definition 2.9. We denote by $W^{u,+}$ the connected component of $W^{u} \backslash O$ that is accumulated (via the Inclination Lemma and the Strong $\lambda$ Lemma [dMP, D]) by the positive orbit of $I_{0}^{+}$. We denote $W^{u,-}$ the connected component of $W_{X}^{u}(O) \backslash O$ accumulated by the positive orbit of $I_{0}^{-}$.

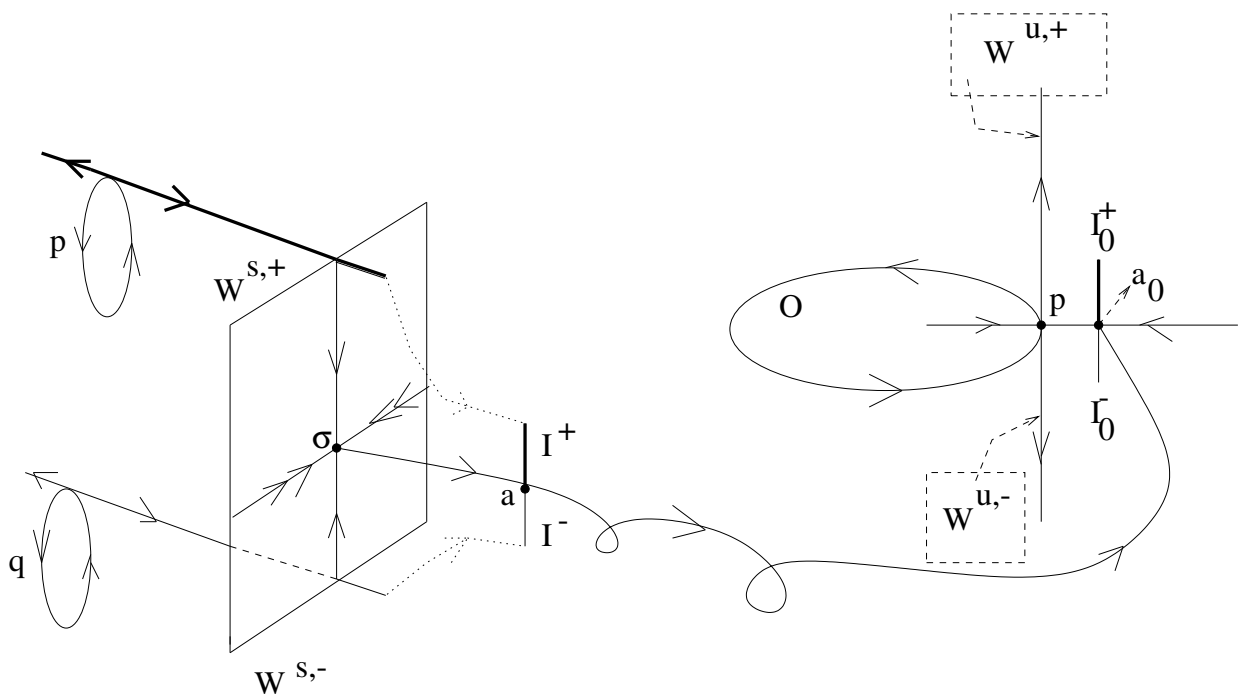

Figure 1. Definition of $W^{u,+}$ and $W^{u,-}$.

It can easily be proved using the Strong $\lambda$ Lemma $[\mathbf{D}]$ that this definition does not depend on $p, q, J_{p}, J_{q}$.

Proposition 2.10. $W^{u,+} \cap W_{X}^{s,-}(\sigma)=\emptyset$ and $W^{u,+} \cap W_{X}^{s,+}(\sigma) \neq \emptyset$. The same is true interchanging + and - .

Proof. For simplicity set $W=W^{u,+}$. First we prove that $W \cap W_{X}^{s,-}(\sigma)=\emptyset$. Suppose for a contradiction that $W \cap W_{X}^{s,-}(\sigma) \neq \emptyset$. Since this last intersection is transversal, there is an interval $J \subset W_{X}^{s,-}(\sigma)$ intersecting $W$ transversally. Now, fix a cross-section $\Sigma=\Sigma^{+}$as in Lemma 2.2 and let $p \in \operatorname{Per}_{X}(\Lambda)$ be such that $W_{X}^{u}(p) \cap W_{X}^{s,+}(\sigma) \neq \emptyset$. Then there is an small interval $I \subset W_{X}^{u}(p) \cap \Sigma$ transversal to $\Sigma \cap W_{X}^{s,+}(\sigma)$. By the definition of $W=W^{u,+}$ (Definition 2.9), the positive orbit of $I$ accumulates on $W$. Since $J$ is transversal to $W$ the Inclination Lemma implies that the positive orbit of $I$ intersects $J$. This proves $W_{X}^{u}(p) \cap W_{X}^{s,-}(\sigma) \neq \emptyset$ for all $p \in \operatorname{Per}_{X}(\Lambda)$. It follows that alternative 2 of Proposition 2.5 holds for all $p, q$, which contradicts the nontransitivity of $\Lambda$ and proves that $W \cap W_{X}^{s,-}(\sigma)=\emptyset$, as desired. Now suppose for a contradiction that $W \cap W_{X}^{s,+}(\sigma)=\emptyset$. Since $W \cap W_{X}^{s,-}(\sigma)=\emptyset$ we obtain $W \cap W_{X}^{s}(\sigma)=\emptyset$ (see $\left.[\mathbf{M P P}]\right)$. But the denseness of periodic orbits and the Inclination Lemma imply that $W \cap W_{X}^{s}(\sigma) \neq \emptyset$. 
This is a contradiction, which proves that $W \cap W_{X}^{s,+}(\sigma) \neq \emptyset$. The result follows.

\section{Proposition 2.11. $H^{+}=\mathrm{Cl}\left(W^{u,+}\right)$ and $H^{-}=\mathrm{Cl}\left(W^{u,-}\right)$.}

Proof. Fix $q \in P^{+}$, i.e., $W_{X}^{u}(q) \cap W_{X}^{s,+}(\sigma) \neq \emptyset$. Note that $W^{u,+} \cap W_{X}^{s,+}(\sigma)$ is nonempty by Lemma 2.10. Using $(\mathrm{H} 1)$ and the Inclination Lemma it is not hard to prove that $W^{u,+}$ accumulates on $q$; therefore $H^{+} \subset \mathrm{Cl}\left(W^{u,+}\right)$. Conversely let $x \in W^{u,+}$ be fixed. By (H1) and $W^{u,+} \subset \Lambda$ there is $z$ in $\operatorname{Per}_{X}(\Lambda)$ near $x$. Choosing $z$ close to $x$ we ensure that $W_{X}^{s}(z) \cap W^{u,+} \neq \emptyset$, because stable manifolds have size uniformly bounded away from zero. If $W_{X}^{u}(z) \cap W_{X}^{s,-}(\sigma) \neq \emptyset$, the Inclination Lemma and the fact that $W_{X}^{s}(z)$ intersects $W^{u,+}$ imply that $W^{u} \cap W_{X}^{s,-}(\sigma) \neq \emptyset$. This contradicts Proposition 2.10, so $W_{X}^{u}(z) \cap W_{X}^{s,-}(\sigma)=\emptyset$. By [MP1] we obtain $z \in P^{+}$, proving that $x \in H^{+}$and the lemma.

Proposition 2.12. If $z \in \operatorname{Per}_{X}(\Lambda)$ and $W_{X}^{s}(z) \cap W^{u,+} \neq \emptyset$, then

$$
\mathrm{Cl}\left(W_{X}^{s}(z) \cap W^{u,+}\right)=\mathrm{Cl}\left(W^{u,+}\right) .
$$

The same is true if we replace + by -

Proof. We have shown that $\mathrm{Cl}\left(W_{X}^{s,+}(\sigma) \cap W^{u,+}\right)=\mathrm{Cl}\left(W^{u,+}\right)$. Fix $x \in$ $W^{u,+}$. By $(\mathrm{H} 1)$ there is $w \in \operatorname{Per}_{X}(\Lambda)$ close to $x$. In particular $W_{X}^{s}(w)$ intersects $W^{u,+}$. If $W_{X}^{u}(w) \cap W_{X}^{s,+}(\sigma)=\emptyset$ then $W_{X}^{u}(w) \cap W_{X}^{s,-}(\sigma) \neq \emptyset$ by [MP1]. It follows from the Inclination Lemma that $W^{u,+} \cap W_{X}^{s,-}(\sigma) \neq \emptyset$, contradicting Proposition 2.10. We conclude that $W_{X}^{u}(w) \cap W_{X}^{s,+}(\sigma) \neq \emptyset$. Note that $W_{X}^{s}(w) \cap W^{u,+} \neq \emptyset$ gets close to $x$ as $w \rightarrow x$. Since the intersection $W_{X}^{u}(w) \cap W_{X}^{s,+}(\sigma) \neq \emptyset$ is transversal we can apply the Inclination Lemma to find a transverse intersection $W^{u,+} \cap W_{X}^{s,+}(\sigma)$ close to $x$. This proves $\mathrm{Cl}\left(W_{X}^{s,+}(\sigma) \cap W^{u,+}\right)=\mathrm{Cl}\left(W^{u,+}\right)$. Finally we prove $\mathrm{Cl}\left(W_{X}^{s}(z) \cap W^{u,+}\right)=$ $\mathrm{Cl}\left(W^{u,+}\right)$. Choose $x \in W^{u,+}$. Since $\mathrm{Cl}\left(W_{X}^{s,+}(\sigma) \cap W^{u,+}\right)=\mathrm{Cl}\left(W^{u,+}\right)$ there is an interval $I_{x} \subset W^{u,+}$ arbitrarily close to $x$ such that $I_{x} \cap W_{X}^{s,+}(\sigma) \neq \emptyset$. The positive orbit of $I_{x}$ first passes through $a$ and then accumulates on $W^{u,+}$. But $W_{X}^{s}(z) \cap W^{u,+} \neq \emptyset$ by assumption. Since this intersection is transversal the Inclination Lemma implies that the positive orbit of $I_{x}$ intersects $W_{X}^{s}(z)$. By taking the backward flow of the last intersection we get $W_{X}^{s}(z) \cap I_{x} \neq \emptyset$. This proves the lemma.

Given $z \in \operatorname{Per}_{X}(\Lambda)$, let $H_{X}(z)$ be the homoclinic class associated to $z$.

Proposition 2.13. If $z \in \operatorname{Per}_{X}(\Lambda)$ is close to a point in $W^{u,+}$, then

$$
H_{X}(z)=\mathrm{Cl}\left(W^{u,+}\right) .
$$

The same is true if we replace + by - . 
Proof. Let $z \in \operatorname{Per}_{X}(\Lambda)$ be a point close to one in $W^{u,+}$. It follows from the continuity of the stable manifolds that $W_{X}^{s}(z) \cap W^{u,+} \neq \emptyset$. We claim that $H_{X}(z)=\mathrm{Cl}\left(W^{u,+}\right)$. Indeed $W^{u,+} \cap W_{X}^{s,-}(\sigma)=\emptyset$ by Proposition 2.10. This equality and the Inclination Lemma imply that $W_{X}^{u}(z) \cap W_{X}^{s}(\sigma) \subset W_{X}^{s,+}(\sigma)$. By Proposition 2.12, $W_{X}^{s}(z) \cap W^{u,+}$ is dense in $W^{u,+}$ since $W_{X}^{s}(z) \cap W^{u,+} \neq$ $\emptyset$. Let $\Sigma$ be a cross-section containing $p_{0}$ and fix $x \in W^{u,+}$. We can assume $x, z \in \Sigma$. Since $W_{X}^{u}(z) \cap W_{X}^{s}(\sigma) \neq \emptyset$ and $W_{X}^{u}(z) \cap W_{X}^{s}(\sigma) \subset W_{X}^{s,+}(\sigma)$ there is an interval $I \subset W_{X}^{u}(z)$ intersecting $W_{X}^{s,+}(\sigma)$. Then the positive orbit of $I$ yields an interval $J$ close to $\sigma$ intersecting $W_{X}^{s,+}(\sigma)$. In addition, the positive orbit of $J$ yields an interval $K$ whose positive orbit accumulates $W^{u,+}$ (recall Definition 2.9). Since $W_{X}^{s}(z) \cap W^{u,+}$ is dense in $W^{u,+}$ and $x \in W^{u,+}$, the orbit $W_{X}^{s}(z)$ passes close to $x$. The Inclination Lemma applied to the positive orbit of $K$ yields a homoclinic point $z^{\prime}$ associated to $z$ which is close to $x$. This proves that $x \in H_{X}(z)$, so $\mathrm{Cl}\left(W^{u,+}\right) \subset H_{X}(z)$. The opposite inclusion is a direct consequence of the Inclination Lemma applied to $W_{X}^{s}(z) \cap W^{u,+} \neq \emptyset$. We conclude that $\mathrm{Cl}\left(W^{u,+}\right)=H_{X}(z)$ as desired.

Theorem 2.14. Let $\Lambda$ be a singular-hyperbolic set of $a C^{r}$ flow $X$ on a closed three-manifold, where $r \geq 1$. Suppose that the following properties hold:

1. $\Lambda$ is connected.

2. $\Lambda$ is attracting.

3. The closed orbits contained in $\Lambda$ are dense in $\Lambda$.

4. $\Lambda$ has a unique singularity $\sigma$.

5. $\Lambda$ is not transitive.

Then $\mathrm{H}^{+}$and $\mathrm{H}^{-}$are homoclinic classes of $X$.

Proof. Let $\Lambda$ be a singular-hyperbolic set of $X$ satisfying the theorem's conditions. To prove that $\mathrm{H}^{+}$is a homoclinic class it suffices by Proposition 2.11 to prove that $\mathrm{Cl}\left(W^{u,+}\right)$ is a homoclinic class. By condition 3 of the Theorem we can choose $z \in \operatorname{Per}_{X}(\Lambda)$ arbitrarily close to a point in $W^{u,+}$. Then $\mathrm{Cl}\left(W^{u,+}\right)=H_{X}(z)$ by Proposition 2.13 and the result follows.

\section{Proof of Theorem A}

First we introduce some notations. Hereafter $M$ is a compact 3-manifold and $\mathcal{X}^{r}$ is the space of $C^{r}$ flows in $M$ equipped with the $C^{r}$ topology, $r \geq 1$. The nonwandering set of $X \in \mathcal{X}^{r}$ is the set $\Omega(X)$ of points $p \in M$ such that for all neighborhood $U$ of $p$ and $T>0$ there is $t>T$ such that $X_{t}(U) \cap U \neq \emptyset$. An attracting $\Lambda$ with isolating block $U$ has a continuation $\Lambda(Y)$ for $Y C^{r}$ close to $X$ defined by $\Lambda(Y)=\bigcap_{t>0} Y_{t}(U)$. This continuation is then defined when $\Lambda$ is an attractor. A compact invariant set is nontrivial if it is not a 
closed orbit of $X$. Transitive sets for flows are always connected. The proof of Theorem A is based on the following result:

Theorem 3.1. Let $\Lambda$ be a singular-hyperbolic set of $X \in \mathcal{X}^{r}, r \geq 1$. Suppose that the following properties hold:

1. $\Lambda$ is connected.

2. $\Lambda$ is attracting.

3. The closed orbits contained in $\Lambda$ are dense in $\Lambda$.

4. $\Lambda$ has only one singularity.

5. $\Lambda$ is not transitive.

Then for every neighborhood $U$ of $\Lambda$ there is a flow $Y$ that is $C^{r}$ close to $X$ and such that

$$
\Lambda(Y) \not \subset \Omega(Y) .
$$

To prove this theorem we shall use the following definitions and facts: let $X \in \mathcal{X}^{r}$ and let $\Lambda$ be a singular-hyperbolic set of $X$ satisfying the conditions of the theorem. Let $\sigma$ be the unique singularity of $\Lambda$. As mentioned on page 330, $\sigma$ is Lorenz-like. As in Section $2, W_{X}^{s s}(\sigma)$ divides $W_{X}^{s}(\sigma)$ into two connected components, which we denote by $W_{X}^{s,+}(\sigma)$ and $W_{X}^{s,-}(\sigma)$, or $W^{s,+}, W^{s,-}$ for short. Recall that $\operatorname{Per}_{X}(\Lambda)$ denotes the union of the periodic orbits of $X$ in $\Lambda$. Fix such $a \in W_{X}^{u}(\sigma) \backslash\{\sigma\}$. By Theorem 2.8, $\omega_{X}(a)=O$ for some periodic orbit with positive expanding eigenvalues of $X$. In particular, $W^{u,+}$ and $W^{u,-}$ are defined (Definition 2.9).

Lemma 3.2. $\mathrm{Cl}\left(W^{u,+}\right) \cap W^{s,-}=\emptyset$.

Proof. Suppose for a contradiction that $\mathrm{Cl}\left(W^{u,+}\right) \cap W^{s,-} \neq \emptyset$. By Lemma 2.2 there is a singular cross-section $\Sigma^{-}$such that every orbit of $\Lambda$ passing close to some point in $W_{X}^{s,-}(\sigma)$ intersects $\Sigma^{-}$. Let $q \in \Lambda$ be periodic such that $W_{X}^{u}(q) \cap W_{X}^{s,-}(\sigma) \neq \emptyset$. Since $\operatorname{Cl}\left(W^{u,+}\right) \cap W^{s,-} \neq \emptyset$, we have $\mathrm{Cl}\left(W^{u,+}\right) \cap \Sigma^{-} \neq \emptyset$. Because closed orbits are dense we can prove that $q \in \mathrm{Cl}\left(W^{u,+}\right)$. It follows that $H^{-} \subset \mathrm{Cl}\left(W^{u,+}\right)$, so $\Lambda=\mathrm{Cl}\left(W^{u,+}\right)$ by Lemma 2.1. Also, since $\Lambda$ is not transitive, $H^{+}=\mathrm{Cl}\left(W^{u,+}\right)$ (Proposition 2.11) and $H^{+}$is a homoclinic class (Theorem 2.14). Since homoclinic classes are transitive sets we conclude that $\Lambda$ is transitive, a contradiction. This proves the result.

Lemma 3.3. Let $D$ be a fundamental domain of $W_{X}^{u u}\left(p_{0}\right)$ contained in $W^{u,+}$. There exist a neighborhood $V$ of $D$ and a cross-section $\Sigma^{-}$of $X$ intersecting $W^{s,-}$ satisfying the following properties:

1. Every $X$-orbit's sequence in $\Lambda$ converging to a point in $W^{s,-}$ intersects $\Sigma^{-}$.

2. No positive $X$-orbit with initial point in $V$ intersects $\Sigma^{-}$.

Proof. Fix a fundamental domain $F$ of $W_{X}^{s}(\sigma)$ and define $F^{-}=F \cap W_{X}^{s,-}(\sigma)$ Then there is a compact interval $F^{\prime} \subset F^{-}$such that $\Lambda \cap F^{-} \subset F^{\prime}$. By 
Lemma 3.2 there is $\epsilon>0$ such that $B_{\epsilon}\left(\mathrm{Cl}\left(W^{u,+}\right)\right) \cap B_{\epsilon}\left(F^{\prime}\right)=\emptyset$. Clearly we can choose a cross-section $\Sigma^{-}$of $X$ inside $B_{\epsilon}\left(F^{\prime}\right)$ such that every $X$-orbit's sequence in $\Lambda$ converging to some point in $W^{s,-}$ intersects $\Sigma^{-}$. Since $W^{u,+}$ is invariant and $\mathrm{Cl}\left(W^{u,+}\right) \cap B_{\epsilon}\left(F^{\prime}\right)=\emptyset$, every positive orbit with initial point in $D$ cannot intersect $\Sigma^{-}$. By using the contracting foliation of $\Lambda$ we have the same property for every positive trajectory with initial point in a neighborhood $V$ of $D$. This proves the result.

Now we define a perturbation (pushing) close to a point $a \in W_{X}^{u}(\sigma) \backslash\{\sigma\}$. To this end we fix the following cross-sections:

1. $\Sigma_{a}$, containing $a$ in its interior.

2. $\Sigma^{\prime}=X_{1}(\Sigma)$.

3. $\Sigma_{0}$, intersecting $O$ in a single interior point.

4. $\Sigma^{+}, \Sigma^{-}$, which intersect $W^{s,+}, W^{s,-}$, respectively, and point toward the side of $a$.

Every $X$-orbit intersecting $\Sigma^{+} \cup \Sigma^{-}$will intersect $\Sigma$. Note that there is a well-defined neighborhood $\mathcal{O}$ given by

$$
\mathcal{O}=X_{[0,1]}\left(\Sigma_{a}\right)
$$

This neighborhood will be the support of the pushing described in the Figures 2 and 3 . The pushing in $\mathcal{O}$ yielding the perturbed flow $Y$ of $X$ is obtained in the standard way (see $[\mathbf{d M P}]$ ).

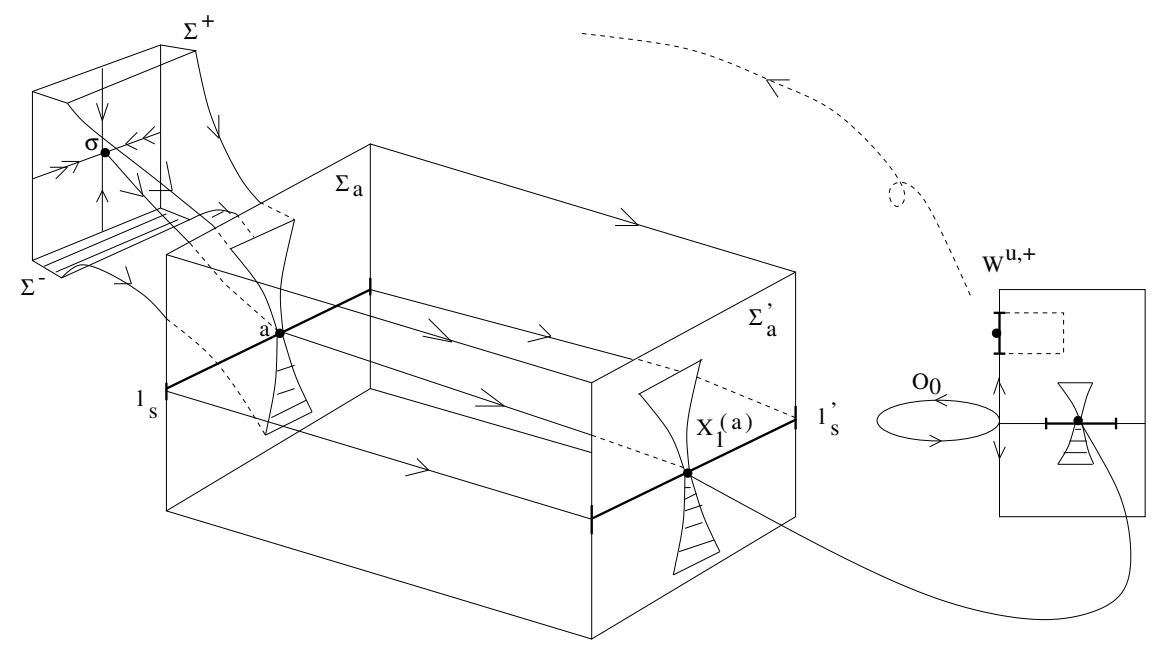

Figure 2. Unperturbed flow $X$.

We have to prove that $\Lambda(Y) \not \subset \Omega(Y)$ for the perturbed flow $Y$. For this purpose we observe that by 5 of Theorem 3.1 and Proposition 2.5 we can assume that there $q$ periodic in $U$ such that $W_{X}^{u}(q) \cap W_{X}^{s,-}(\sigma) \neq \emptyset$. We obtain in this way an interval $K$ in $\Sigma^{-} \cap W_{X}^{u}(q)$ crossing $\Sigma^{-}$as in 


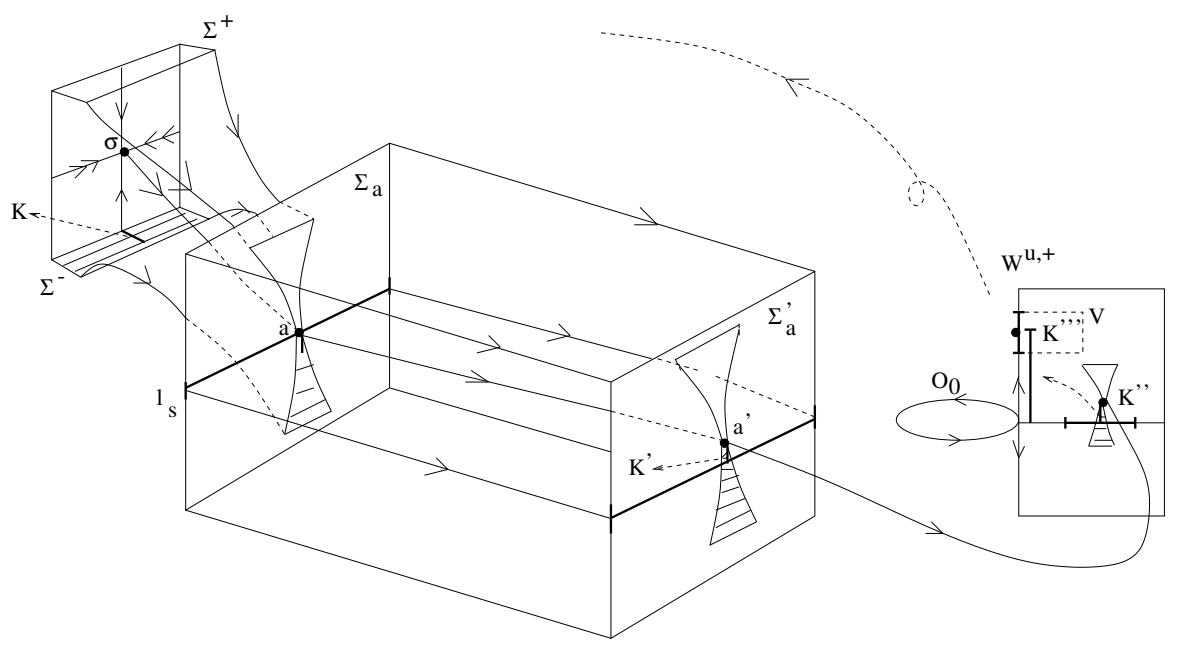

Figure 3. Perturbed flow $Y$.

Figure 3. The $Y$-flow carries $K$ to an interval $K^{\prime \prime}$ as in Figure 3. Let $q(Y), W_{Y}^{u}(q(Y)), K^{\prime \prime}(Y), \sigma(Y)$ denote the continuation of these objects for the perturbed flow $Y$. We observe that $K(Y) \subset \Lambda(Y)$ since $\Lambda(Y)$ is an attracting set, $q(Y) \in \Lambda(Y)$ and $K \subset W_{Y}^{u}(q(Y))$. Then Theorem 3.1 will follow from the lemma below:

\section{Lemma 3.4. $K(Y) \not \subset \Omega(Y)$.}

Proof. Suppose for a contradiction that $K(Y) \subset \Omega(Y)$ and pick $x \in \operatorname{Int} K(Y)$, the interior of the interval $K(Y)$. The flow of $Y$ carries the points near $x$ to the neighborhood $V$ depicted in Figure 3. This neighborhood is obtained by saturating a fundamental domain in $W^{u,+}$ by the strong stable manifolds [HPS]. Note that there are points $x^{\prime}$ near $x$ that back up close to $x$ under the forward flow of $Y(x \in K(Y) \subset \Omega(Y))$. In particular, the positive $Y$ orbit of $x^{\prime}$ returns to $\Sigma^{-}$. At the same time, Lemma 3.3-2 implies that no $X$-orbit starting in $V$ intersects $\Sigma^{-}$. Since $X=Y$ outside $\mathcal{O}$ we conclude that the positive $Y$-orbit of $x^{\prime}$ intersects $\Sigma^{+}$. Afterward this positive orbit passes through the box $\mathcal{O}$ and arrives to $V$. By repeating the argument we conclude that the positive $Y$-orbit of $x^{\prime}$ never returns to $\Sigma^{-}$, a contradiction. The lemma is proved.

Proof of Theorem A. Let $\Lambda$ be a singular-hyperbolic attractor of a $C^{r}$ flow $X$ on a compact 3-manifold $M$. Assume that $\Lambda$ is $C^{r}$ critically robust and has a unique singularity $\sigma$. Denote by $\Lambda(Y)=\bigcap_{t>0} Y_{t}(U)$ the continuation of $\Lambda$ in a neighborhood $U$ of $\Lambda$ for $Y$ close to $X$. Denote by $C(Y)$ the union of the closed orbits of a flow $Y$. Since $\Lambda$ is $C^{r}$ critically robust, there is a neighborhood $U$ of $\Lambda$ such that $\Lambda(Y) \cap C(Y)$ is dense in $\Lambda(Y)$ for every flow 
$Y$ that is $C^{r}$ close to $X$. Clearly $\Lambda(Y)$ is a singular-hyperbolic set of $Y$ for all $Y$ close to $X$. Because $\Lambda$ has a unique singularity, so does $\Lambda(Y)$. Now recall that $\Lambda$ is an attractor by assumption. In particular, $\Lambda$ is transitive (recall Definition 1.1). It follows that $\Lambda$ is connected and so the neighborhood $U$ above can be arranged to be connected. Then $\Lambda(Y)$ is connected as well. Summarizing, $\Lambda(Y)$ is a singular-hyperbolic set of $Y$ satisfying conditions 1-4 of Theorem 3.1. If $\Lambda$ is not $C^{r}$ robust, we can find a $Y$ that is is $C^{r}$ close to $X$ and such that $\Lambda(Y)$ is not transitive. Then $\Lambda(Y)$ satisfies all conditions of Theorem 3.1, and we can find a $Y^{\prime}$ that is $C^{r}$ close to $Y$ and such that $\Lambda\left(Y^{\prime}\right) \not \subset \Omega\left(Y^{\prime}\right)$. This is a contradiction, since $\Lambda\left(Y^{\prime}\right) \subset \Omega\left(Y^{\prime}\right)$ (recall that $\Lambda\left(Y^{\prime}\right) \cap C\left(Y^{\prime}\right)$ is dense in $\left.\Lambda\left(Y^{\prime}\right)\right)$. This contradiction proves the result.

\section{References}

[B] C. Bonatti, $C^{1}$-generic dynamics: tame and wild behavior, Proceedings of the International Congress of Mathematicians, Vol. III (Beijing, 2002), 265-277, Higher Ed. Press, Beijing, 2002, MR 1957538 (2003k:37002).

[Bw] R. Bowen, Symbolic dynamics for hyperbolic flows, Amer. J. Math., 95 (1973), 429-460, MR 0339281 (49 \#4041), Zbl 0282.58009.

[dMP] W. de Melo and J. Palis, Geometric Theory of Dynamical Systems. An Introduction, Translated from the Portuguese by A. K. Manning, Springer-Verlag, New York-Berlin, 1982, MR 0669541 (84a:58004), Zbl 0491.58001.

[D] B. Deng, The Silnikov problem, exponential expansion, strong $\lambda$-lemma, $C^{1}$ linearization, and homoclinic bifurcation, J. Differential Equations, 79 (1989), 189-231, MR 1000687 (90k:58161), Zbl 0674.34040.

[GW] J. Guckenheimer and R.F. Williams, Structural stability of Lorenz attractors, Inst. Hautes Études Sci. Publ. Math., 50 (1979), 59-72, MR 0556582 (82b:58055a), Zbl 0436.58018.

[HPS] M. Hirsch, C. Pugh and M. Shub, Invariant Manifolds, Lectures Notes in Mathematics 583, Springer, Berlin, 1977, MR 0501173 (58 \#18595), Zbl 0355.58009.

[Mi] J. Milnor, On the concept of attractor, Comm. Math. Phys., 99 (1985), 177195, MR 0790735 (87i:58109a), Zbl 0595.58028; Correction and remarks: "On the concept of attractor", Comm. Math. Phys. 102 (1985), 517-519, MR 0818833 (87i:58109b), Zbl 0602.58030.

[M] C.A. Morales, Singular-hyperbolic sets and topological dimension, Dyn. Syst., 18(2) (2003), 181-189, MR 1994788 (2004g:37037).

[MP1] C.A. Morales and M.J. Pacifico, Mixing attractors for 3-flows, Nonlinearity, 14 (2001), 359-378, MR 1819802 (2002a:37036), Zbl 1026.37015.

[MP2] C.A. Morales and M.J. Pacifico, Transitivity and homoclinic classes for singularhyperbolic systems, preprint, 2003, to appear.

[MPP] C.A. Morales, M.J. Pacifico and E.R. Pujals, On $C^{1}$ robust transitive sets for three-dimensional flows, C.R. Acad. Sci. Paris, Série I (Math.), 326 (1998), 81-86, MR 1649489 (99j:58183), Zbl 0918.58036.

$[\mathrm{MPu}]$ C.A. Morales and E.R. Pujals, Singular strange attractors on the boundary of Morse-Smale systems, Ann. Sci. Ecole Norm. Sup., 30 (1997), 693-717, MR 1476293 (98k:58137), Zbl 0911.58022. 
[PT] J. Palis and F. Takens, Hyperbolicity and Sensitive Chaotic Dynamics at Homoclinic Bifurcations. Fractal Dimensions and Infinitely Many Attractors, Cambridge Studies in Advanced Mathematics, 35, Cambridge University Press, Cambridge, 1993, MR 1237641 (94h:58129), Zbl 0790.58014.

$[\mathrm{P}] \quad$ E.R. Pujals, Tangent bundle dynamics and its consequences, Proceedings of the International Congress of Mathematicians, Vol. III (Beijing, 2002), 327-338, Higher Ed. Press, Beijing, 2002, MR 1957543 (2004c:37036).

Received April 24, 2003. The work was partially supported by CNPq, FAPERJ and PRONEX/DYN. SYS.

Instituto DE MATEMÁticA

Universidade Federal do Rio DE JANEIRO

C.P. 68.530

21945-970 Rio DE JANEIRO, RJ

BRAZIL

E-mail address: morales@impa.br

Instituto DE MATEMÁtica

Universidade Federal do Rio DE JANEIRo

C.P. 68.530

21945-970 Rio de Janeiro, RJ

BRAZIL

E-mail address: pacifico@impa.br 